\title{
Circulating miRNA Expression Profiling and Target Prediction in Patients Receiving Dexmedetomidine
}

\author{
Xuehui Yang ${ }^{\mathrm{a}}$ Hongmei Chen $^{\mathrm{b}}$ Yan Chen $^{\mathrm{b}} \quad$ Yochai Birnbaum $^{\mathrm{c}}$ \\ Rongbi Liang ${ }^{\mathrm{a}} \quad$ Yumei Ye $^{\mathrm{d}}$ Jinqiao Qian ${ }^{\mathrm{a}}$ \\ aDepartment of Anesthesiology, First Affiliated Hospital of Kunming Medical University, Kunming, \\ 'Department of Anesthesiology, Kunming Angel Women's \& Children's Hospital, Kunming, China, \\ 'Department of Medicine, Section of Cardiology, Baylor College of Medicine, Houston, dDepartment of \\ Biochemistry and Molecular Biology, University of Texas Medical Branch, Galveston, USA
}

\section{Key Words}

Dexmedetomidine $\cdot$ Circulating miRNAs $•$ Expression profiling Ischemia/reperfusion injury • Cardioprotection

\begin{abstract}
Background/Aims: Circulating miRNAs could serve as biomarkers for diagnosis or prognosis of heart diseases and cerebrovascular diseases. Dexmedetomidine has protective effects in various organs. The effects of dexmedetomidine on circulating miRNAs remain unknown. Here, we investigated differentially expressed miRNA and to predict the target genes of the miRNA in patients receiving dexmedetomidine. Methods: The expression levels of circulating miRNAs of 3 patients were determined through high through-put miRNA sequencing technology. Target genes of the identified differentially expressed miRNAs were predicted using TargetScan 7.1 and miRDB v.5. Gene Ontology (GO) and the Kyoto Encyclopedia of Genes and Genomes (KEGG) were used to conduct functional annotation and pathway enrichment analysis of target genes respectively. Results: Twelve differentially expressed miRNAs were identified. Five miRNAs were upregulated (hsa-miR-4508, hsa-miR-novel-chr8_87373, hsa-miR-30a3p, hsa-miR-novel-chr16_26099, hsa-miR-4306) and seven miRNAs (hsa-miR-744-5p, hsamiR-320a, hsa-miR-novel-chr9_90035, hsa-miR-101-3p, hsa-miR-150-5p, hsa-miR-342-3p, and hsa-miR-140-3p) were downregulated after administration of dexmedetomidine in the subjects. The target genes and pathways related to the differentially expressed miRNAs were predicted and analyzed. Conclusion: The differentially expressed miRNAs may be involved in the mechanisms of action of dexmedetomidine. Specific miRNAs, such as hsa-miR-101-3p, hsa-miR-150-5p and hsa-miR-140-3p, are new potential targets for further functional studies of dexmedetomidine.

$\mathrm{X}$. Yang and $\mathrm{H}$. Chen contributed equally to this work.

\begin{tabular}{ll}
\hline Jinqiao Qian & Department of Anesthesiology, First Affiliated Hospital of Kunming Medical University \\
& \#295 Xichang Road, Kunming, Yunnan Province 650032 (China) \\
& E-Mail qianjinqiao@126.com
\end{tabular}




\section{Cellular Physiology Cell Physiol Biochem 2018;50:552-568 \begin{tabular}{ll|l} 
DOI: 10.1159/000494168 & O 2018 The Author(s). Published by S. Karger AG, Basel \\
www.karger.com/cpb
\end{tabular}}

Yang et al.: Dexmedetomidine and Circulating miRNA

\section{Introduction}

miRNAs are a class of endogenous, small ( $\sim 22$ nucleotides [nt]), noncoding singlestranded RNAs [1]. miRNAs regulate genes involved in a range of diverse biological processes, including angiogenesis, development, stress response, inflammation, adhesion, proliferation, differentiation, and apoptosis [2, 3].

miRNAs were initially found in intracellular locations, so most studies have assessed the miRNAs expression in original tissue samples. An amazing discovery revealed that miRNAs is found in extracellular spaces, such as urine, blood, and other bodily fluids, where they are stable, despite the presence of RNase [4]. Extracellular miRNAs were firstly reported in cancer patients, and since then they have been extensively studied for their potential functions and as markers of diseases [5, 6].. Extracellular miRNAs, which are excreted through various mechanisms that are not yet fully understood, may be protected from degradation by several mechanisms, including their inclusion in microvesicles/exosomes and apoptotic bodies. Recent studies have found that miRNAs are actively secreted into microvesicles or exosomes of various cell types [4, 7]. miRNAs may also be directly released from damaged cells or through apoptotic bodies in atherosclerosis or myocardial infarction [8]. In addition, miRNAs may also be protected from degradation in the extracellular space by forming specific protein-miRNA complexes that resist RNase activity [7].

Over the past few years, several studies have suggested circulating miRNAs as possible biomarkers for the diagnosis or prognosis of heart diseases (coronary artery disease, myocardial infarction, hypertension, heart failure, angiogenesis, atrial fibrillation, cardiomyopathy, dyslipidemia), and cerebrovascular disease (stroke) [9].

Dexmedetomidine is a selective and potent $\alpha 2$-adrenergic receptor agonist, and was approved by the US Food and Drug Administration in 1999 for procedural sedation of patients in intensive care units. Dexmedetomidine has several beneficial actions during the perioperative period. It attenuates sympathetic tone, with reduction of the neuroendocrine and hemodynamic responses to anesthesia and surgery; decrease opioid and anesthetic requirements; and causes sedation without risk of respiratory depression. Many reports have noted other possible applications including use as a protective agent against ischemia/ reperfusion $(I / R)$ injury in various organs [10, 11], including heart [12], kidney [13], brain [14], lung [15], liver [16], and even retina [17]. Only three studies have addressed the roles of miRNAs in mechanisms of organ protection $[14,18,19]$. Moreover, no clinical trials have focused on miRNA expression profiling after administration of dexmedetomidine, and there are no studies on the effects of dexmedetomidine on circulating miRNAs.

The aims of the study were to identify via a high-throughput approach the differentially expressed miRNAs in blood samples from patients receiving dexmedetomidine, and to conduct bioinformatics analysis to determine the potential functions of the differentially expressed miRNAs and possible mechanisms of action of dexmedetomidine through Gene Ontology (GO) and Kyoto Encyclopedia of Genes and Genomes (KEGG) pathway analyses, respectively.

\section{Materials and Methods}

\section{Subject and sample collection}

Subjects were patients scheduled for elective procedures who had the American Society of Anesthesiologists (ASA) I or II physical status and were 20-65 years of age. Exclusion criteria were the following: a body mass index $>35 \mathrm{~kg} / \mathrm{m}^{2}$, hepatic, renal or cardiac insufficiency, hypovolemia, bradycardia, bundle branch block, atrioventricular block, diabetes, pregnant, breast-feeding or menstruating women, alcohol or drug abuse, psychiatric disease, allergy or contraindication to dexmedetomidine. We randomly enrolled 8 patients scheduled to undergo surgery with a 1-month period. Of them, 5 were excluded 


\section{Cellular Physiology Cell Physiol Biochem 2018;50:552-568 \begin{tabular}{l|l|l} 
and Biochemistry $10.1159 / 000494168$ & $\begin{array}{l}\text { () 2018 The Author(s). Published by S. Karger AG, Basel } \\
\text { www.karger.com/cpb }\end{array}$
\end{tabular}}

Yang et al.: Dexmedetomidine and Circulating miRNA

according to the above criteria, leaving 3 for this study who underwent arthroscopic repair for rotator cuff tears, radical esophagectomy for esophageal cancer via video-assisted thoracic surgery, and resection of hepatic hemangioma, respectively.

The arterial lines were established under local anesthesia (2\% lidocaine) for invasive blood pressure monitoring. The patients were administered an initial loading dose $(1 \mu \mathrm{g} / \mathrm{kg})$ of dexmedetomidine over 10 $\mathrm{min}$ followed by a maintenance dose $(1 \mu \mathrm{g} / \mathrm{kg} / \mathrm{h})$ for $20 \mathrm{~min}$. They did not receive any other medication or sedatives before and during administration of dexmedetomidine. The hemodynamic parameters (heart rate and blood pressure) and blood oxygen saturation $\left(\mathrm{SpO}_{2}\right)$ were recorded before and 1, 5, 10 and 30 minutes after administration of dexmedetomidine. Blood samples were collected before and 30 minutes after administration of dexmedetomidine (control samples and test samples).

Written informed consent was obtained from all three participants. All procedures in this study were approved by Ethnics Committees of First Affiliated Hospital of Kunming Medical University and conformed to the principles outline in the Declaration of Helsinki.

\section{RNA isolation and quality control}

The purity and concentrations of total RNA of the blood samples were measured with NanoDrop ND1000. The inclusion criterion for the RNA was an OD of A260/A280 ratio between 1.8 and 2.1.

\section{miRNA sequencing}

Total RNA of each sample was used to prepare the miRNA sequencing library, through the following steps: (1) 3'-adaptor ligation. (2) 5'-adaptor ligation. (3) cDNA synthesis. (4) PCR amplification. (5) size selection of $\sim 135-155$ bp PCR-amplified fragments (corresponding to $\sim 15-35 \mathrm{nt}$ small RNAs).The libraries were denatured as single-stranded DNA molecules, captured on Illumina flow cells(Illumina, San Diego, CA), amplified in situ as clusters and finally sequenced for 51 cycles on Illumina NextSeq per the manufacturer's instructions.

\section{Extraction of miRNA and small RNA sequencing}

Six samples were analyzed. Total RNA was isolated using Trizol reagent (Invitrogen, Carlsbad, CA, USA). Small RNA sequencing was performed by KangChen Bio-tech (Shanghai, China) using the Illumina Small RNA Sequencing Platform. The TruSeq small RNA library preparation kit (Illumina) was used for library preparation. Sequencing was performed using an Illumina HiSeq 2000 sequencing system, and 10 $\mathrm{Mb}$ of clean reads were analyzed using routine algorithms

\section{Bioinformatics analysis}

The GO project provides a controlled vocabulary to describe gene and gene product attributes in any organism (http://www.geneontology.org). The ontology covers three domains: biological process, cellular component and molecular function. Fisher's exact test was used to determine if there is more overlap between the differentially expressed list and the GO annotation list than would be expected by chance. The $P$-value denotes the significance of GO terms enrichment in the differentially expressed genes. The lower the $P$-value, the more significant the GO term $(P$-value $<0.05$ is recommended).

Pathway analysis is a functional analysis mapping genes to KEGG pathways. The $P$-value (EASEscore, Fisher- $P$ value or hypergeometric- $P$ value) denotes the significance of the pathway correlated to the conditions. Lower the p-value, more significant is the pathway (the recommend $P$-value cutoff is 0.05 ).

Two databases were used to predict miRNAs' target genes: TargetScan7.1 and miRDB v5 (http:// mirdb.org/miRDB).TargetScan7.1 was used for human and mouse (human miRNA targets are from http:// www.targetscan.org/vert_71/, mouse miRNA targets are from http://www.targetscan.org/mmu_71/).

\section{Statistical analysis}

Results are expressed as means \pm SE. Data were compared using paired $t$-test for hemodynamic parameters. Values of $P<0.05$ were considered statistically significant $(P<0.1$ for differentially expressed miRNAs).

When comparing the differentially expressed miRNA profiles between two groups, fold change and $P$-value were calculated and used to identify significant significantly differentially expressed miRNAs (based on all-isoform value). Differentially expressed miRNAs between two samples were filtered through 
fold change (based on all-isoform value), followed by hierarchical clustering. miRNA target prediction was performed by TargetScan7.1 and miRDB v5, and then the GO and KEGG pathway analyses were performed based on the top 10 differentially expressed miRNAs.

\section{Results}

Changes in hemodynamic parameters following administration of dexmedetomidine

Heart rate decreased significantly following administration of dexmedetomidine compared with baselines $(P<0.05)$, but none of the patients required treatment. Blood pressure was decreased following administration of dexmedetomidine, but there were no significant differences compared with baselines and no patients required administration of vasopressors. There was no change in $\mathrm{SpO}_{2}$ before and after administration of dexmedetomidine (Table 1)

\section{Quality control}

The RNA A260/A280 ratios were 1.8 and 1.9. For quality assessment of the sequencing library, an Agilent 2100 Bioanalyzer and Agilent DNA 1000 chip kit were used according to the manufacturer's protocols. All quality criteria for successful miRNA sequencing were fulfilled.

\section{Cluster analysis of miRNA sequencing data}

Pearson's correlation coefficient $\mathrm{R}$ for the test samples and control samples showed a high degree of correlation $(R=0.9984$; Fig. 1). miRNA sequencing identified 12 miRNAs with significantly differential expression (fold change $>1$.2-fold, $P<0.1$ ) after administration of dexmedetomidine in the subjects. Five miRNAs were upregulated (hsa-miR-4508, hsamiR-novel-chr8_87373, hsa-miR-30a-3p, hsa-miR-novel-chr16_26099, hsa-miR-4306) and 7 miRNAs were downregulated (hsa-miR-744-5p, hsa-miR-320a, hsa-miR-novelchr9_90035, hsa-miR-101-3p, hsamiR-150-5p, hsa-miR-342-3p, and hsa-miR-140-3p) (Table 2). After filtering low-intensity miRNAs, raw signal intensities were normalized by median. The differentially expressed miRNAs passed volcano

Table 1. Changes in hemodynamic parameters after administration of dexmedetomidine $(\mathrm{n}=3, \quad \overline{\mathrm{x}} \pm \mathrm{se}) * \mathrm{P}<0.05$ compared with baseline

\begin{tabular}{lccccc}
\hline Parameter & Baseline & $1 \mathrm{~min}$ & $5 \mathrm{~min}$ & $10 \mathrm{~min}$ & $30 \mathrm{~min}$ \\
\hline $\mathrm{HR}(\mathrm{BPM})$ & $74.67 \pm 3.33$ & $66.00 \pm 4.00^{*}$ & $57.33 \pm 3.93^{*}$ & $50.67 \pm 2.96^{*}$ & $51.67 \pm 2.03^{*}$ \\
$\mathrm{SBP}(\mathrm{mmHg})$ & $139.33 \pm 6.36$ & $134.33 \pm 2.33$ & $132.00 \pm 3.06$ & $135.67 \pm 9.21$ & $127.67 \pm 1.67$ \\
$\mathrm{DBP}(\mathrm{mmHg} H$ & $80.67 \pm 3.48$ & $72.33 \pm 1.67$ & $70.33 \pm 2.03$ & $73.00 \pm 5.03$ & $71.00 \pm 2.00$ \\
$\mathrm{MAP}(\mathrm{mmHg})$ & $100.33 \pm 3.84$ & $92.67 \pm 1.86$ & $90.67 \pm 2.33$ & $94.00 \pm 6.56$ & $90.00 \pm 2.00$ \\
$\mathrm{SpO}_{2}(\%)$ & $100 \pm 0$ & $100 \pm 0$ & $100 \pm 0$ & $100 \pm 0$ & $100 \pm 0$ \\
\hline
\end{tabular}

Fig. 1. The plots of differentially expressed microRNAs. A. Pearson's correlation coefficient $\mathrm{R}$ for serum microRNAs before and after administration of dexmedetomidine showing a high degree of correlation $(\mathrm{R}=0.9984)$. $\mathrm{B}$. volcano plot of differentially expressed microRNAs from serum of patients receiving dexmedetomidine (Test vs Control). The vertical lines correspond to 1.2-fold up and down, respectively, and the horizontal line represents a

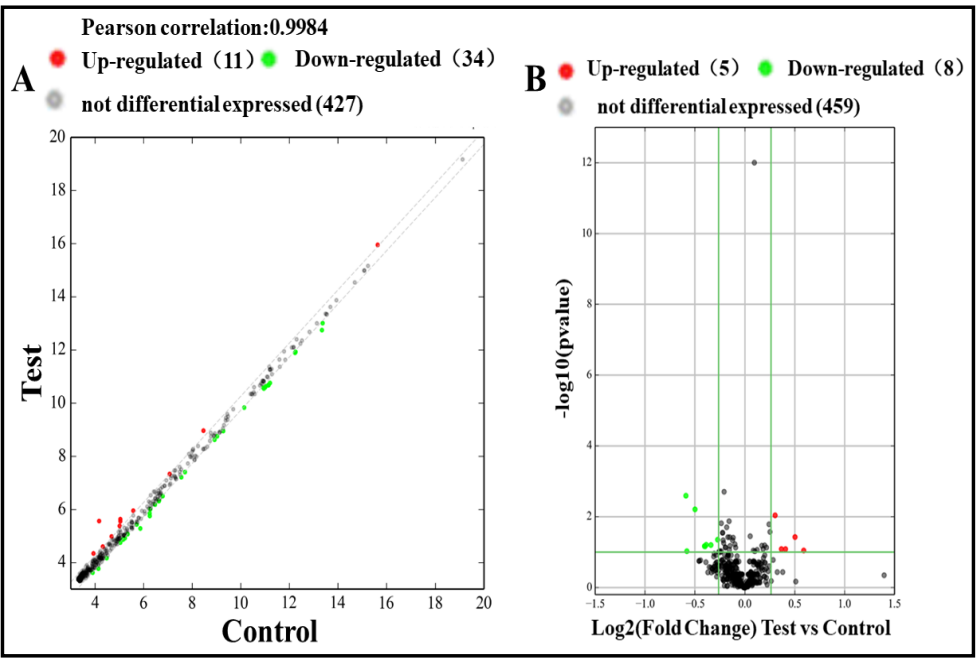
$\mathrm{p}$-value of 0.1 . So the red and the green points in the plot represent the differentially expressed microRNAs with significant difference. 
plot filtering (fold change $>1.2$-fold, $P$-value $<0.1$; Fig. 1). Unsupervised hierarchic clustering was performed based on the 12 differentially expressed miRNAs and displayed as a heat map (Fig. 2).

TargetScan7.1 and miRDB v5 were used to predict the targets of differentially expressed miRNA in the blood samples, and we found that each miRNA had multiple predicted target genes. Two Venn diagrams were generated to highlight the relationship between the two databases. There are 232 overlapping genes for the downregulated miRNAs (Table 3) and 173 overlapping genes for the upregulated miRNAs (Table 4) identified by the two databases, which are most likely to be targets of the differentially expressed miRNAs before and after administration of dexmedetomidine (Fig. 3).

\section{GO and KEGG pathway analyses}

GO was used to classify the function of up- and downregulated genes from 3 structured networks: biological processes, cellular components, and molecular function. In this study, differentially expressed mRNAs were enriched in numerous biological processes including: homophilic cell adhesion via plasma membrane adhesion molecules, cell-cell adhesion via plasma-membrane adhesion molecules, regulation of macromolecule metabolic process, regulation of metabolic process, regulation of primary metabolic process, regulation of nucleobasecontaining compound metabolic process, regulation of nitrogen compound metabolic process, nervous system development,

Table 2. Differentially expressed miRNAs in serum of patients after administration of dexmedetomidine

\begin{tabular}{lcccc}
\hline Mature-ID & Fold change & P value & Regulation & FDR \\
\hline hsa-miR-4508 & 1.505051 & 0.089855421 & Up & 0.747156078 \\
hsa-miR-novel-chr8_87373 & 1.416824 & 0.037418429 & Up & 0.747156078 \\
hsa-miR-30a-3p & 1.326087 & 0.082014908 & Up & 0.747156078 \\
hsa-miR-novel-chr16_26099 & 1.28866 & 0.08184383 & Up & 0.747156078 \\
hsa-miR-4306 & 1.233766 & 0.009132611 & Up & 0.747156078 \\
hsa-miR-744-5p & 0.827869 & 0.044466914 & Down & 0.747156078 \\
hsa-miR-320a & 0.789998 & 0.062857532 & Down & 0.747156078 \\
hsa-miR-novel-chr9_90035 & 0.764715 & 0.061976551 & Down & 0.747156078 \\
hsa-miR-101-3p & 0.75865 & 0.068637291 & Down & 0.747156078 \\
hsa-miR-150-5p & 0.707424 & 0.00617971 & Down & 0.747156078 \\
hsa-miR-342-3p & 0.668571 & 0.093971171 & Down & 0.40074689 \\
hsa-miR-140-3p & 0.664249 & 0.00254712 & Down & 0.747156078 \\
\hline
\end{tabular}

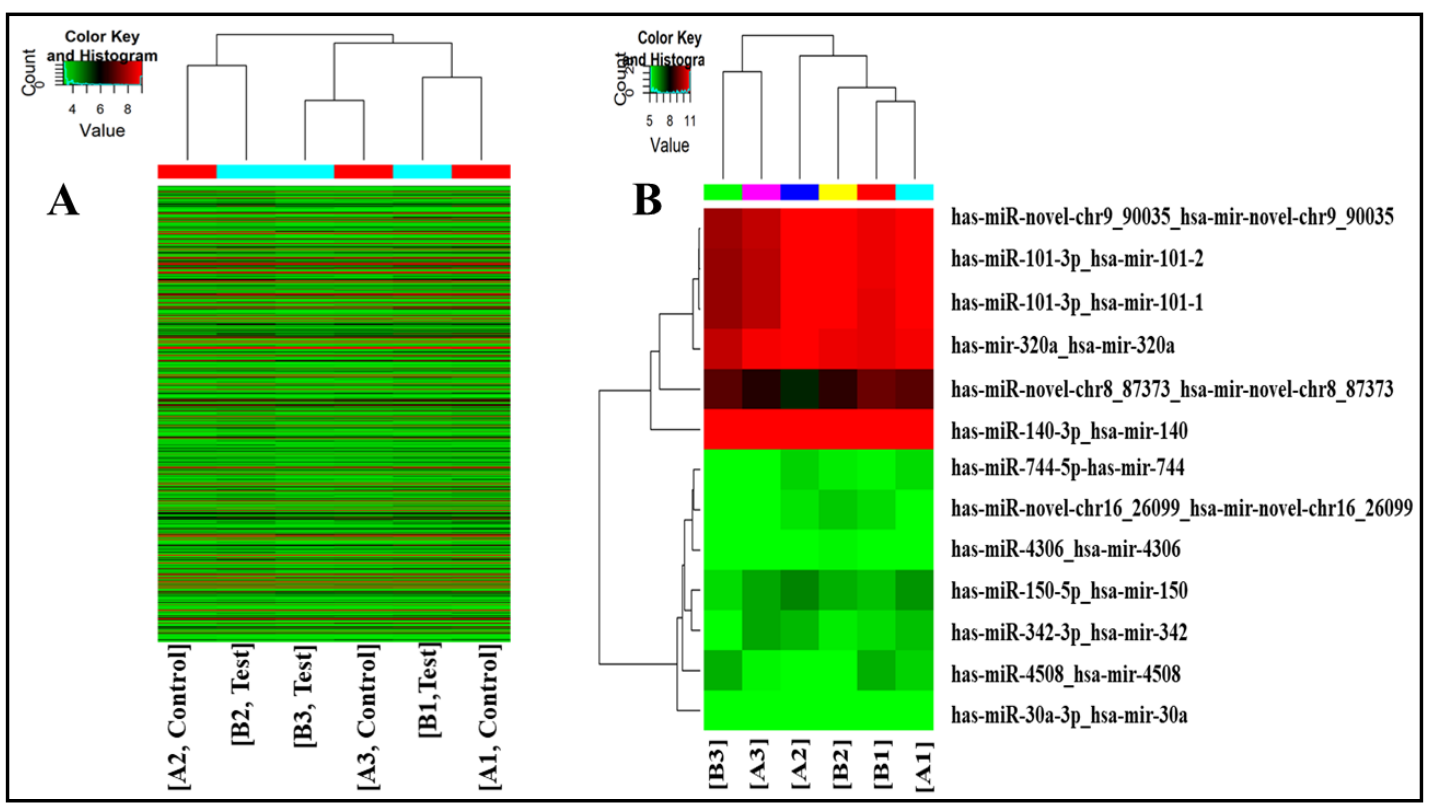

Fig. 2. The hierarchical clustering analysis of differentially expressed microRNAs. Samples names are listed at the bottom. The names of the significantly $(\mathrm{p}<0.1)$ expressed microRNAs are shown on the right. Thirteen microRNAs were expressed differently in target genes analysis. Color gradation shows the relative expression of microRNAs before and after administration of dexmedetomidine: green, downregulation; red, upregulation. A. heatmap for all expression; B. heatmap for Test vs Control. 
Table 3. Target prediction of downregulated miRNAs: the intersection of mirdbV5 and TargetScan7.1

\begin{tabular}{|c|c|}
\hline miRNA name & Gene symbol \\
\hline & SLTSCR1, CACNB2, STAMBP, CDH5, FZD6, RAB1A, SULT4A1, FOS, TGFBR1, SCN8A, RANBI \\
\hline & \\
\hline & CCI1, \\
\hline \multirow[t]{4}{*}{ hsa-miR-101-3p } & ZMAT3, ZFP36L2, CERS6, RAC1, PNISR, LMNB1, PCDH8, SLC12A2, MRGBP, GLRA2, MBNL1, TET2, PTGS2, ZFAND3, MOB4, SMARCA1, HTRA3, UBE \\
\hline & 5 K1C, TSHZ3, CAV3, SEL1L, GAB1, COL10A1, ANKRD11, NLK, NDFIP1, RIN2, ARNTL2, PAPOLG, PPTC7, EMP2, SMARCD1, \\
\hline & $\begin{array}{l}\text { ARHGEF3, PRKAA1, ASPN, SYNCRIP, SLC39A10, FAM60A, MFSD6, EZH2, ZNF654, MAML3, DAG1, R } 7 \\
\text { CPEB3 PRKCE KBTBD8 PRPF4B H2AFV RORA, SHSA6 ABHD17C RASD2 MYRIP NC }\end{array}$ \\
\hline & \\
\hline & WTAP, HILPDA, MYB, ZMAT2, GRIPA \\
\hline hsa-mir & PCDH \\
\hline & $\begin{array}{l}\text { TMEM255A, COPS2, GNA11, AP3M1, PCDHA3, LPPR1, CNKSR2, PCDHA4, KCNS3, PCDHA12, DNER, MSI2, HECTD2, CD K6, PCDHA7, PAPD5, YOD 1, ARPP19, RASA1, SYNGR2, } \\
\text { RBM24, DESI2, PCDHA2, PCDHA1, RAI2, DPY30, PCDHAC1, PCDHAC2, PBX3, SDHD, PCDHA5, RGS9BP, KITLG, PCDHA6, PCDHA13 }\end{array}$ \\
\hline & OSER1, UBE2D2, MMS19, FUT8, DTNBP1, FAM53C, SYNP02L, MRFAP1, ID4, GXY \\
\hline hsa-miR-744-5p & 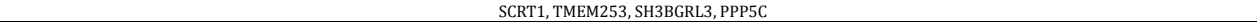 \\
\hline
\end{tabular}

Table 4. Target prediction of upregulated miRNAs: the intersection of miRDB v.5 and TargetScan7.1

\begin{tabular}{|c|c|}
\hline miRNA name & Gene symbol \\
\hline hsa-miR-30a-3p & $\begin{array}{l}\text { RFK, CDKN1B, YPEL5, SLC35F3, PDK1, ARF6, RYR3, ZNHIT6, TROVE2, C2orf66, RPAP3, WDR44, GFPT1, FAM134B, SLM02, NOX3, ARMC1, SERBP1, HLA- } \\
\text { B, TPK1, ELL3, UNG, YTHDF3, PSMD10, EIF1, SDHAF2, ZNF22, WFDC8, SLC25A33, PHLDA1, HIRA, PANK3, C9orf170, KIAA1324L, STAU1, CDC14A, ZNF24, } \\
\text { SIAH1, ZNF138, USP6NL, HSDL1, GREM1, TMEM106B, ATG12, C6orf211, UBE2G1, SBNO1, COLEC12, ADA, APC, CNPY2, RAB33B, GNPNAT1, ITGA1, } \\
\text { NPY2R, CAMK2G, SH3GLB1, DNAJB14, RANBP3L, SCAF4, GPR137C, PTEN, GOLM1 }\end{array}$ \\
\hline hsa-miR-4306 & $\begin{array}{l}\text { SIX3, PCDHA8, CNTD1, CCND2, IKZF4, EIF4B, PCDHA6, TRIM44, CAMK2D, ATP6V1F, ZC3H11A, CDC42, ZNF385A, VIPAS39, PCDHA4, EIF5A, PCDHAC1, } \\
\text { PCDHAC2, SMG7, PCDHA12, PCDHA11, PAK6, CTSK, SGMS1, PBX2, PCDHA2, USH1C, URM1, NFATC3, DPF2, MDM4, ATP1A3, PHF7, PCDHA1, SOX13, } \\
\text { DCTN3, THRA, SLC25A53, RAB35, SPIN3, DYNLL2, NMNAT2, PCDHA7, PCDHA5, KIF3C, DLG2, TOX3, SNX30, PCDHA13, DNM1, SF1, GPR26, SLC39A14, } \\
\text { FAIM2, SLC25A15, CA10, ANKRD34A, TRIM5, SLC16A2, SPATA2, PCDHA10, FAM76A, PCDHA3, TANC2, PCDHA9, TEAD1, LCOR, FAM101A, BSN, CDH4, }\end{array}$ \\
\hline hsa-miR-4508 & $\begin{array}{l}\text { XYLT1, GPR61, CAPZB } \\
\text { PRRG2, LYPLA2, SYT7, SLC43A3, TBX1, VANGL2, RAE1, SLC7A5, SH3GL1, H2AFY, RRP7A, KCNAB3, PPDPF, CXCR1, MORN1, CC2D1A, RBFOX3, VPS37D, } \\
\text { SALL3, DLX2, IL10RA, TREM1, ODF3L2, BRD9, ANXA11, BOK, ATG4B, SMG7, BRE, CXorf36, LRP3, C15orf32, } \\
\text { ARHGAP39, EMD, ADD1, EPS8L3, NTN5 }\end{array}$ \\
\hline
\end{tabular}

Fig. 3. Venn diagrams shows the number of overlap target genes of differentially expressed microRNAs. A. the overlap of upregulated differentially expressed microRNAs; B. the overlap of downregulated differentially expressed microRNAs.
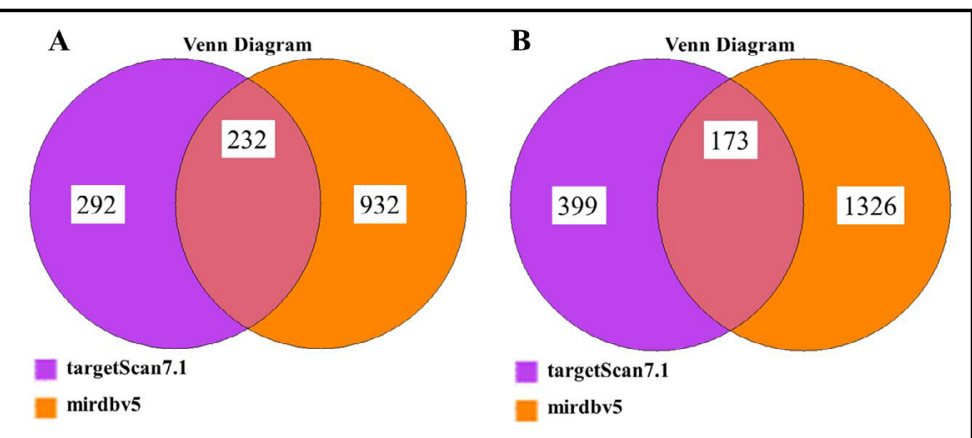

regulation of cellular metabolic process, and chromatin modification (Fig. 4). Similarly, the following cellular components were affected: transferase complex, nucleus, ESC/E (Z) complex, nuclear part, nuclear lumen, intracellular membrane-bounded organelle, nucleoplasm part, catalytic complex, methyltransferase complex, and nucleoplasm (Fig. 5). Affected molecular functions include the following: calcium ion binding, potassium channel regulator activity, binding, protein binding, channel regulator activity, GDP binding, histone methyltransferase activity, RNA stem-loop binding, enzyme binding, transcription factor activity, and transcription factor binding (Fig. 6).

Moreover, KEGG pathway analysis identified the following significantly affected pathways for downregulated miRNAs: MAPK signaling pathway, pathways in cancer, leukocyte transendothelial migration, sphingolipid signaling pathway, bacterial invasion of epithelial cells, transcriptional misregulation in cancer, cocaine addiction, regulation of lipolysis in adipocytes, endocytosis, and melanogenesis (Fig. 7). It also identified the following pathways for upregulated miRNAs: endocytosis, Wnt signaling pathway, HIF-1 signaling pathway, p53 signaling pathway, axon guidance, ErbB signaling pathway, glioma, central carbon metabolism in cancer, and epithelial cell signaling in Helicobacter pylori infection and gastric acid secretion (Fig. 8). 


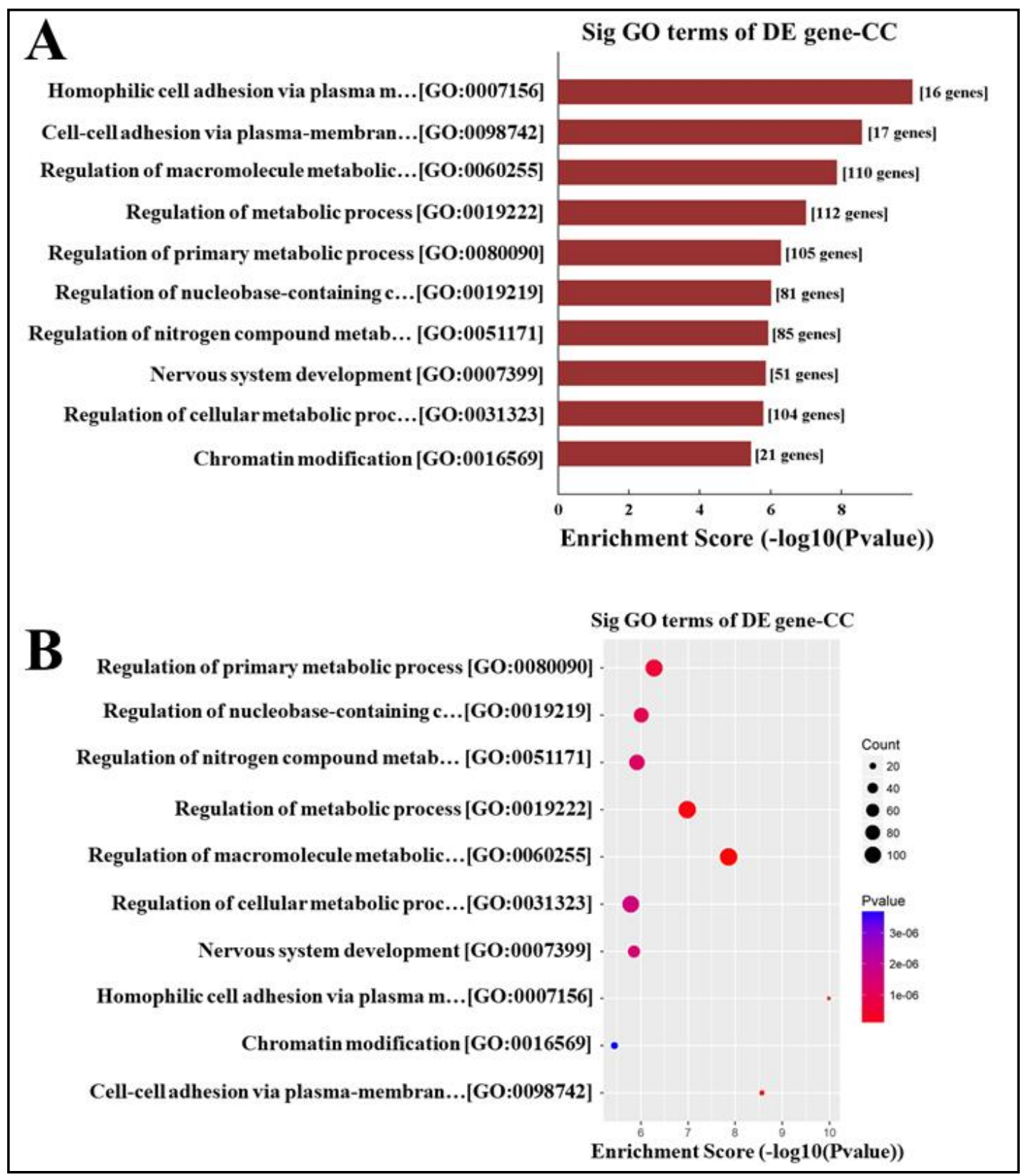

Fig. 4. GO analysis for differentially expressed (DE) mRNA-Biological process (BP). A. Bar plot explanation (Enrichment Score): the bar plot shows the top ten Enrichment Score value of the significant enrichment terms. B. Dot plot explanation (Enrichment Score): The dot plot shows the enrichment score values of the top ten most significant enrichment terms. 


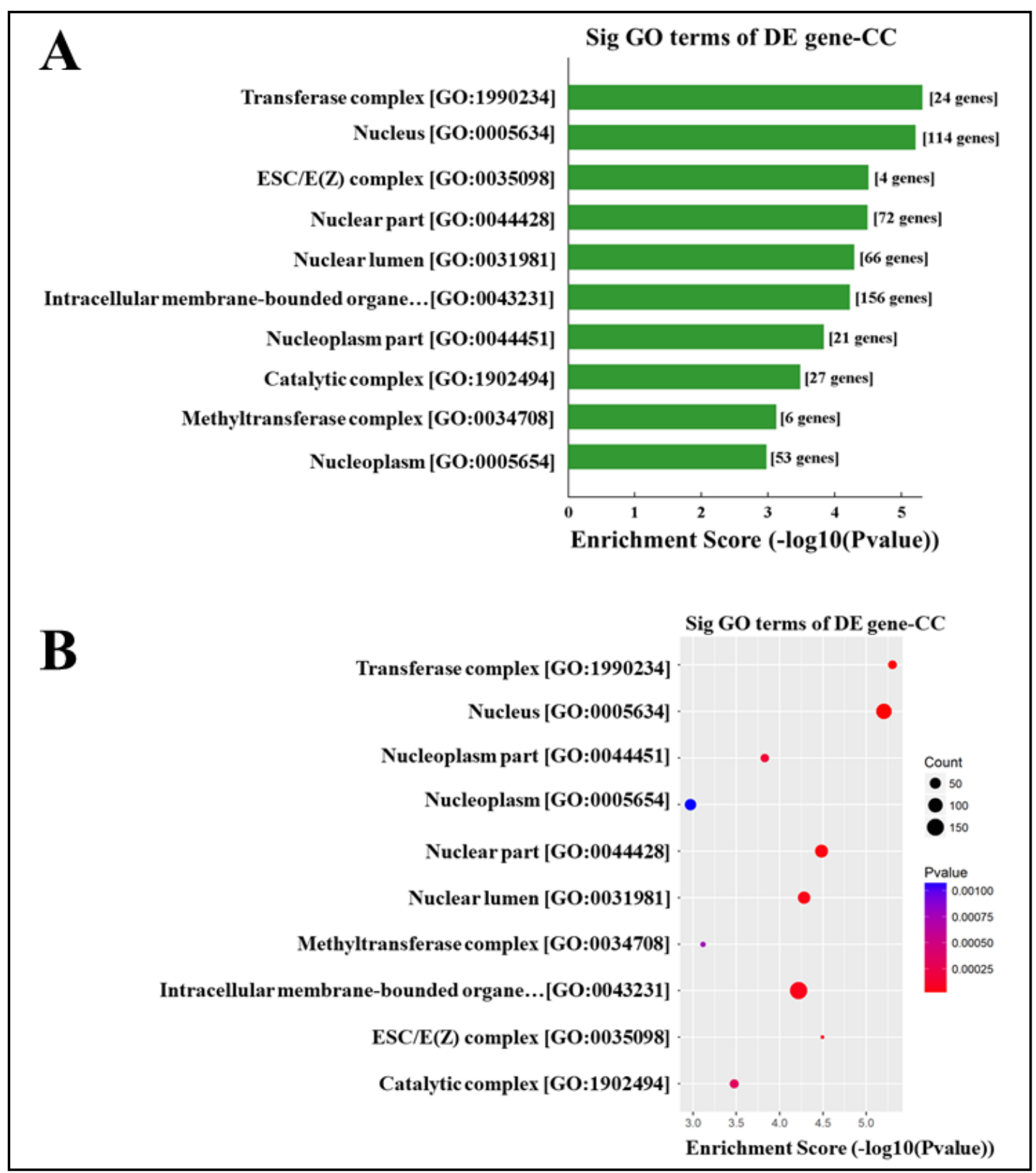

Fig. 5. GO analysis for differentially expressed (DE) mRNA-Cellular component (CC). A. Bar plot explanation (Enrichment Score): the bar plot shows the top ten Enrichment Score value of the significant enrichment terms. B. Dot plot explanation (Enrichment Score): The dot plot shows the enrichment score values of the top ten most significant enrichment terms. 


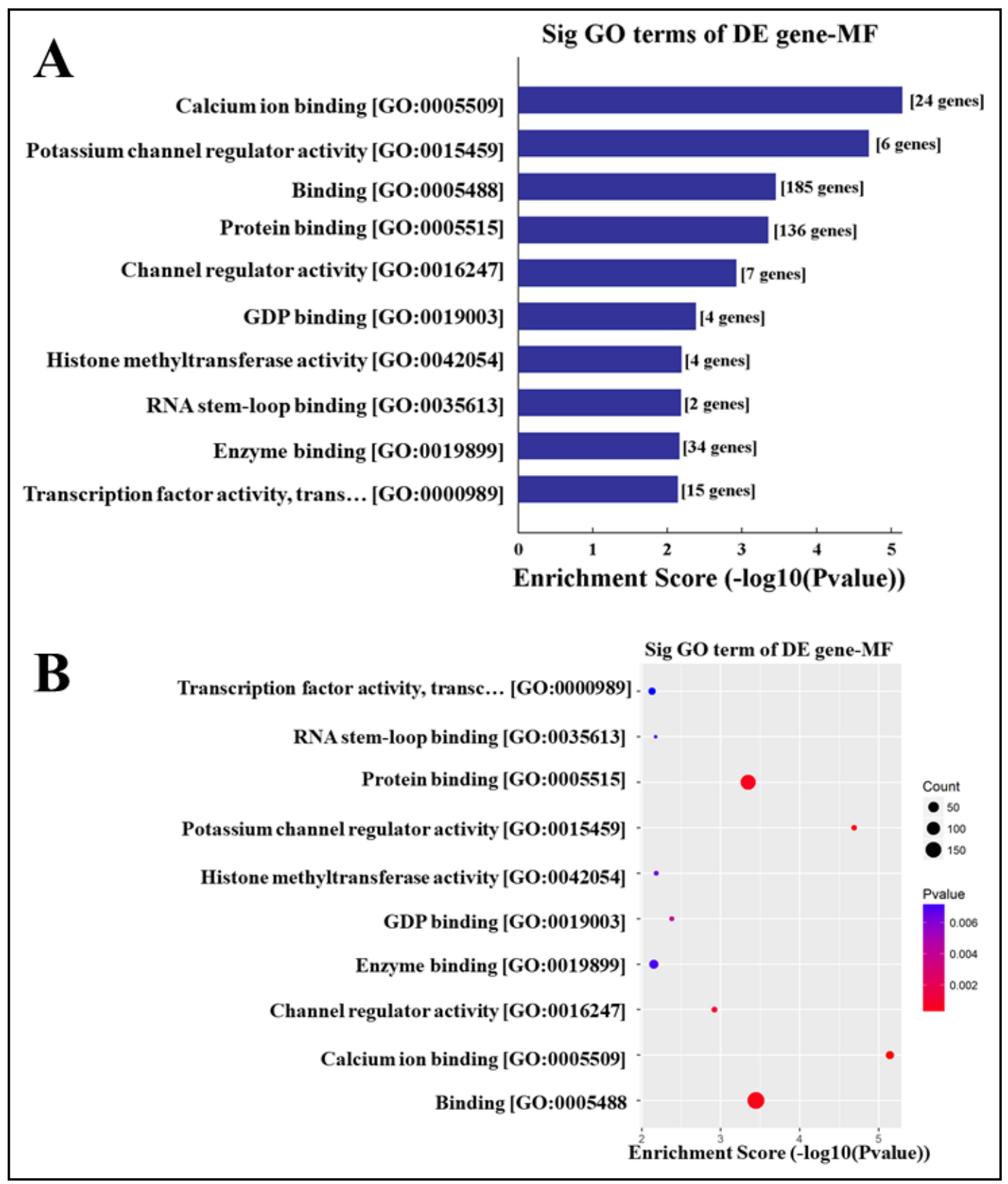

Fig. 6. GO analysis for differentially expressed (DE) mRNA-Molecular function (MF). A. Bar plot explanation (Enrichment Score): the bar plot shows the top ten Enrichment Score value of the significant enrichment terms. B. Dot plot explanation (Enrichment Score): The dot plot shows the enrichment score values of the top ten most significant enrichment terms. 


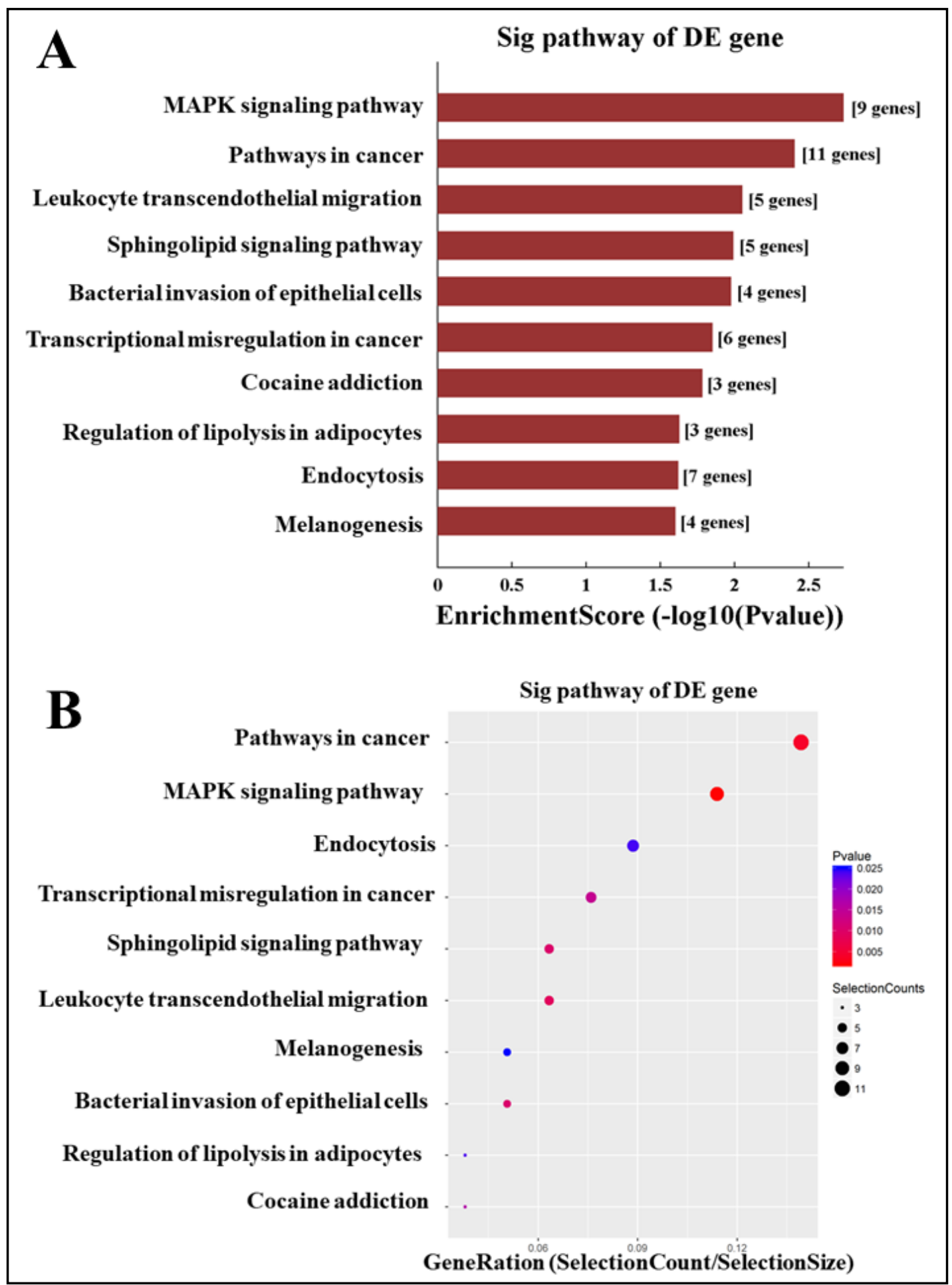

Fig. 7. Pathway analysis of target genes of downregulated microRNAs. Target genes were enriched into different pathways based on KEGG pathway database, the top 10 pathways were shown above. DE: differentially expressed. A. Enrichment Score: the Enrichment Score value of the PathwayID, it equals "-log10 (P value)"; B. GeneRatioDotPlot: The dot plot shows the gene ratio value of the top ten most significant enrichment pathways. 


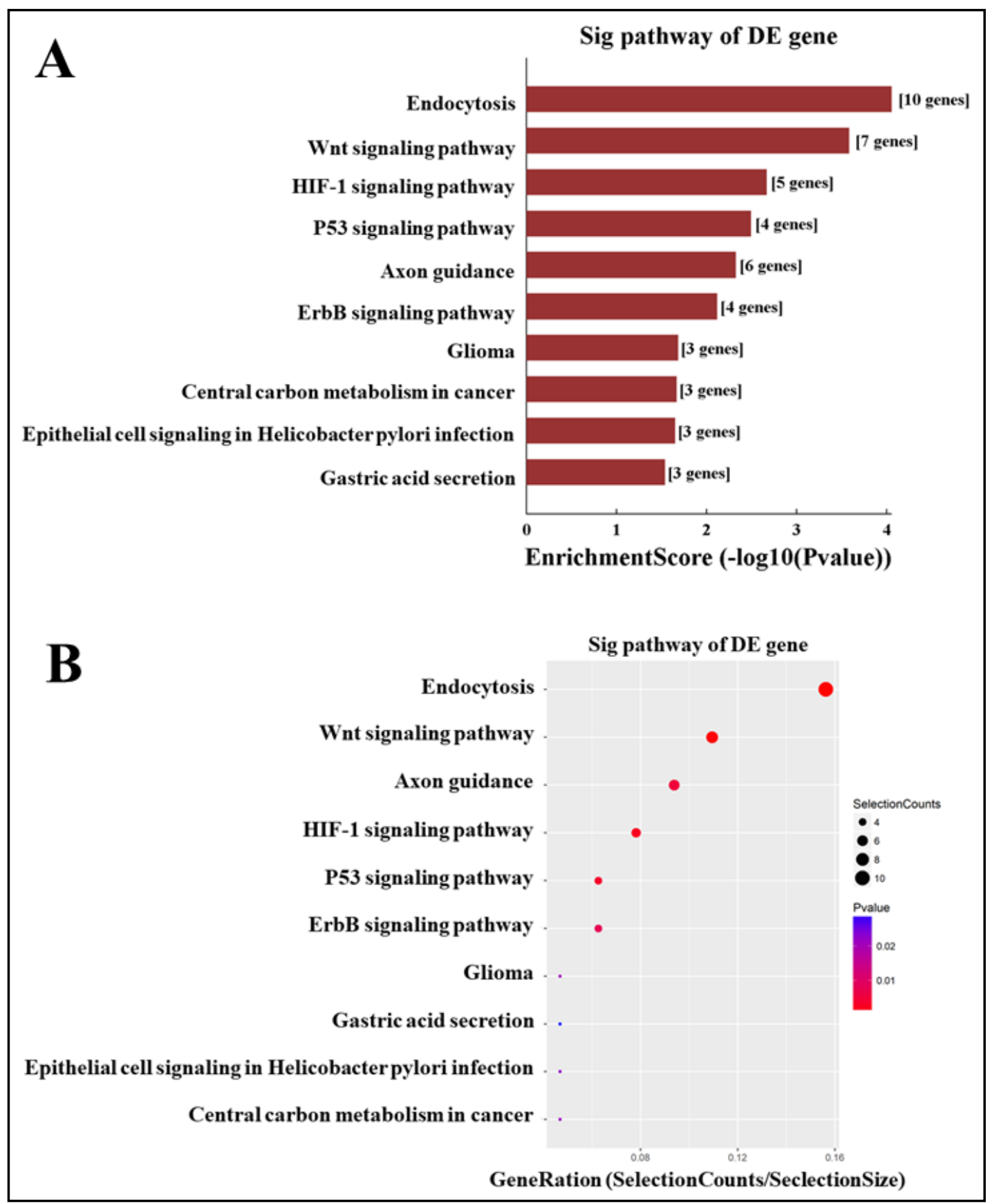

Fig. 8. Pathway analysis of target genes of upregulated microRNAs. Target genes were enriched into different pathways based on KEGG pathway database, the top 10 pathways were shown above. DE: differentially expressed. A. Enrichment Score: the Enrichment Score value of the PathwayID, it equals "-log10 (P value)"; B. GeneRatioDotPlot: The dot plot shows the gene ratio value of the top ten most significant enrichment pathways. 


\section{Cellular Physiology Cell Physiol Biochem 2018;50:552-568 \begin{tabular}{l|l|l} 
and Biochemistry Published 10.1159/000494168 & $\begin{array}{l}\text { (c) } 2018 \text { The Author(s). Published by S. Karger AG, Basel } \\
\text { www.karger.com/cpb }\end{array}$
\end{tabular} \\ Yang et al.: Dexmedetomidine and Circulating miRNA}

\section{Discussion}

The patients had significant bradycardia after receiving dexmedetomidine, which is consistent with the findings of previous studies $[10,11]$. At the same time, blood pressure was slightly decreased, but no significant difference was detected after administration of dexmedetomidine. One possible reason for this discrepancy could be our smaller sample size.

RNA isolation from blood and subsequent quantification by real-time PCR is timeconsuming. Therefore, a more rapid and efficient method is needed to make it feasible to detect circulating miRNAs in clinical practice. With the development of high-throughput platforms including PCR and microarrays, next-generation-sequencing has quickly emerged as the preferred platform for studying circulating miRNAs. Next-generation sequencing has several advantages, including high sensitivity to measure miRNA levels over a wide dynamic range, ability to identify novel miRNAs and to detect miRNA expression levels in species for which complete genomes are not yet available. In addition, next-generation sequencing is able to detect miRNAs that differ by just one nucleotide $[20,21]$. In the present study, we employed high-throughput miRNA sequencing to identify miRNA expression before and after administration of dexmedetomidine in 3 patients and to ascertain that our measurement of miRNAs expression profiling is reliable and accurate.

The findings indicated that 12 differentially expressed miRNA were identified, including 5 upregulated human miRNAs and 7 downregulated human miRNAs, among which, we found 3 novel miRNAs; two of these were upregulated (hsa-miR-novel-chr8_87373 and hsa-miRnovel-chr16_26099), while one was downregulated (hsa-miR-novel-chr9_90035). Until now, no studies to our knowledge have focused on the roles of these 3 novel miRNAs in biology and pathology. Whether or not these 3 novel miRNAs are involved in the protective effects of dexmedetomidine remains unknown.

Upregulation of miR-140 was associated with increased right ventricle systolic pressure and hypertrophy. These results indicate that miR-140 plays a role in the pathogenesis of pulmonary artery hypertension-associated right ventricular dysfunction [22]. Another study found that miR-140-3p was one of upregulated miRNAs in afterload-enhancement-induced pathological hypertrophy in engineered heart tissue [9]. Karakas et al. [23] shows that miR-140-3p derived from peripheral blood predicts mortality, and thus could be a valuable biomarker for risk estimation in coronary artery disease. Recent studies indicate that miR$140-3 p$ is significantly elevated during early stages of acute coronary syndrome [24, 25], and elevated plasma miR-140-3p in patients with acute coronary syndrome mainly originates from circulating endothelial cells and lymphocytes [24]. Li et al. [26] reported that knockdown of miR-140 reduced myocardial infarct sizes in an animal model, whereas miR-140 was upregulated upon apoptotic stimulation. In this study, miR-140-3p was downregulated 30 minutes after administration of dexmedetomidine. This result suggests that downregulation of miR-140-3p by dexmedetomidine may confer cardioprotective effects on myocardial ischemia/reperfusion (I/R) injury.

Scrutinio et al. [27] found miR-150-5p to be significantly dysregulated in patients with advanced heart failure. miR-150-5p is associated with maladaptive remodeling, disease severity, and outcome. miR-150-5p may act as a novel circulating biomarker for advanced heart failure. miR-150 has been previously reported to be upregulated in patients with myocardial infarction [28]. In our study, miR-150-5p was found to be downregulated significantly after administration of dexmedetomidine. Taken together, these results suggest that dexmedetomidine-reduced miR-150 may play an important role in cardioprotective effects of dexmedetomidine.

Circulating hsa-miR-4306 was shown to be elevated in patients with acute stroke, whereas circulating hsa-miR-320e and hsa-miR-320d were reduced in patients compared with healthy individuals [29]. It has been proposed that hsa-miR-4306, hsa-miR-320e and hsa-miR-320d in plasma may serve as novel biomarkers for the early detection of acute stroke in humans [29]. miR-4306 were was shown to be significantly downregulated both in 
plasma and in sclerotic samples of patients with arteriosclerosis obliterans compared with controls [30]. Our study indicates that hsa-miR-4306 in plasma was upregulated, whereas hsa-miR-320a was decreased 30 minutes following administration of dexmedetomidine.

Upregulation of miR-320 has been shown to promote cardiomyocyte death and apoptosis, while downregulation was cytoprotective for simulated I/R injury. Furthermore, overexpression of miR-320 increased apoptosis and infarction size in the hearts on I/R in vivo and ex vivo experiments_ENREF_43. In contrast, in vivo treatment with antagomir-320 reduced infarct size [31]. Both miR-320 and miR-140 are elevated in human with heart failure [32]. miR-320a was identified as a marker to discriminate between patients experiencing and those not experiencing major adverse cardiovascular events within the first year after ST-segment-elevation myocardial infarction [33]. miR-320a was significantly upregulated in heart failure myocardium compared to normal controls [34]. miR-320a can be used to detect patients with systolic heart failure and correlate with clinical prognostic parameters such as dilatation of the left ventricle and left atrium, a wide QRS complex and elevated serum natriuretic peptide levels [35]. miR-320a was recently identified as a potential novel circulating biomarker of arrhythmogenic cardiomyopathy [36]. These studies suggest that differential expression of miR-320a is associated with pathophysiological mechanisms that trigger heart failure, myocardial infarction, and cardiomyopathy. In our study, serum miR320 a was found to be significantly reduced $30 \mathrm{~min}$ after administration of dexmedetomidine. These findings suggests that the administration of dexmedetomidine to patients with heart failure may be beneficial. miR-320 has pro-apoptotic effects on cardiomyocytes; dexmedetomidine may exert cardioprotective effects via reduction of miR-320 expression.

Heart-structure-specific transcriptomic atlas data has shown that miR-744 is cardiac valve specific [37]. Circulating miR-744 was recently reported to be upregulated in patients with chronic congestive heart failure [38]. In the present study, mir-744-5p was found to be decreased after administration of dexmedetomidine. Taken together, these results suggest that patients with heart failure undergoing surgical procedures would benefit from administration of dexmedetomidine intraoperatively.

A recent report indicated that miR-101 inhibits fibrosis and thus may help preserve left ventricle function after myocardial infarction [39] Xiao et al. also reported that upregulation of miR-101a leads reduced fibrosis and scar formation in cardiac tissue from rats with myocardial infarction [40]. Inhibition of miR-101 was shown to attenuate hypoxia/ reoxygenation-induced apoptosis through induction of autophagy in $\mathrm{H} 9 \mathrm{C} 2$ cardiomyocytes [41]. In another study, the level of miR-101-3p was significantly higher in heart transplant recipients with histologically verified acute cellular rejection compared with a control group and could discriminate between patients with and without allograft rejection [42]. In our study, miR-101-3p was downregulated in patients receiving dexmedetomidine.

Through GO functional and KEGG pathway enrichment analyses, the target genes of the differentially expressed miRNAs were those involved in cell apoptosis, metabolism and signal transduction. Therefore, it could be hypothesized that differentially expressed miRNAs may be involved in mechanisms of action of dexmedetomidine via regulation of the target genes via cell apoptosis, metabolism and signal transduction.

Based on bioinformatics analysis, two pathways are probably related to organ protection: MAPK signaling and sphingolipid signaling. Some target genes are involved in MAPK signaling, such as CACNB2, DUSP1, FOS, NLK, PPP5C, RAC1, RAP1B, RASA1 and TGFBR1. Other target genes are associated with sphingolipid signaling such as CERS2, CERS6, GNAI1, PRKCE and RAC1.

It has been reported that dexmedetomidine exerts kidney protection [43] and lung protective effects [44] via inhibition of the MAPK signaling pathway but attenuates neuroapoptosis via activation of this pathway $[45,46]$. In the present study, we found miRNA-101-3p to be significantly downregulated. KEGG analysis suggests that seven of nine genes of the MAPK signaling pathway are target genes of miRNA-101-3p: CACNB2, DUSP1, FOS, NLK, RAC1, RAP1B and TGFBR1. Regulation of the miR-101/MAPK pathway plays an important role in proliferation and metastasis of gallbladder carcinoma [47], in LPS-activated 
macrophages [48], in systemic lupus erythematosus [49] and in proliferation and migration of cardiac fibroblast [50]. Wang et al. found that inhibition of miR-101could attenuate H9C2 apoptosis induced by hydrogen peroxide [41]. Thus, future studies are warranted to elucidate effects of dexmedetomidine on the miR-101/MAPK pathway.

Sphingolipid signaling is involved in protecting various organs, including the heart [51, 52], retina [53], and brain [54]. In the present study, KEGG analysis demonstrated that the sphingolipid signaling pathway could exert effects via CERS2, CERS6, GNAI1, PRKCE, and RAC1 genes, among which, CERS2, CERS6, PRKCE, and RAC1 are overlapping target genes of miR-101-3p, as predicted by the two databases. The remaining gene, GNAI1, is an overlapping target gene of miR-320a, as predicted by the two databases. miR-101-3p shares many genes with the MAPK signaling and sphingolipid signaling pathways; future studies on association of miR-101-3p with these two pathway will be informative and productive.

In sum, our results show that the differentially expressed miRNAs in this study are involved in pathological processes of various cancers, organ protection, and heart failure, but our future researches will focus on the effect of dexmedetomidine on organ I/R injury and heart failure, which frequently occur perioperatively and are problems faced by anesthesiologists. Moreover, this is the first clinical study to identify differential expression of circulating miRNAs following administration of dexmedetomidine, which is a key step to realize translation from bench to bedside.

This study had some limitations. Firstly, the fold-change and $P$-values thresholds were 1.2 and $<0.1$, respectively, which are different from those commonly accepted and were used here because of the relatively short time of exposure to dexmedetomidine. Secondly, some miRNAs are expressed in a time- and stage-specific manner [30]. Thus, the expression pattern of dysregulated miRNAs is not always constant and may fluctuate with time. We did not determine the expression of miRNAs at different time points following administration of dexmedetomidine. Finally, the sample size was relatively small and there was no validation of target genes.

\section{Conclusion}

12 miRNAs were found to be differentially expressed following administration of dexmedetomidine. Furthermore, specific miRNAs, such as miR-140, miR-150-5p, and miR-101-3p, might prove to be important targets for the organ protection. In the future, we will further investigate the functions of these miRNAs to broaden our understanding of mechanisms of action of dexmedetomidine.

\section{Acknowledgements}

This work was supported by the grants from National Natural Science Foundation of China (81460044, 81860050 and 81160035), Yunnan Provincial Science and Technology Department (2014HB031 and 2017FE468 (-039)), Health and Family Planning Commission of Yunnan Province (2018NS0126), Program for Innovative Research Team of Kunming Medical University (CXTD201802) and Yunnan health training project of high level talents.

\section{Disclosure Statement}

The authors declare that no potential competing interests exist. 


\section{Cellular Physiology Cell Physiol Biochem 2018;50:552-568 \begin{tabular}{l|l|l} 
and BOI: 10.1159/000494168 & $\begin{array}{l}\text { (O) 2018 The Author(s). Published by S. Karger AG, Basel } \\
\text { www.karger.com/cpb }\end{array}$
\end{tabular}}

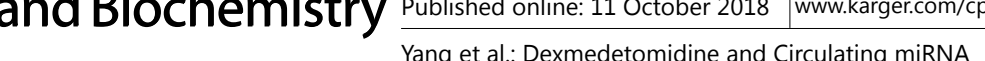

\section{References}

1 Suzuki HI, Miyazono K: Emerging complexity of microRNA generation cascades. J Biochem 2011;149:15-25.

-2 Porrello ER: microRNAs in cardiac development and regeneration. Clin Sci (Lond) 2013;125:151-166.

-3 Condorelli G, Latronico MV, Cavarretta E: microRNAs in cardiovascular diseases: current knowledge and the road ahead. J Am Coll Cardiol 2014;63:2177-2187.

4 Gupta SK, Bang C, Thum T: Circulating microRNAs as biomarkers and potential paracrine mediators of cardiovascular disease. Circ Cardiovasc Genet 2010;3:484-488.

5 Kosaka N, Iguchi H, Ochiya T: Circulating microRNA in body fluid: a new potential biomarker for cancer diagnosis and prognosis. Cancer Sci 2010;101:2087-2092.

-6 Widera C, Gupta SK, Lorenzen JM, Bang C, Bauersachs J, Bethmann K, Kempf T, Wollert KC, Thum T: Diagnostic and prognostic impact of six circulating microRNAs in acute coronary syndrome. J Mol Cell Cardiol 2011;51:872-875.

7 Lorenzen JM, Thum T: Circulating and urinary microRNAs in kidney disease. Clin J Am Soc Nephrol 2012;7:1528-1533.

8 Zernecke A, Bidzhekov K, Noels H, Shagdarsuren E, Gan L, Denecke B, Hristov M, Koppel T, Jahantigh MN, Lutgens E, Wang S, Olson EN, Schober A, Weber C: Delivery of microRNA-126 by apoptotic bodies induces CXCL12-dependent vascular protection. Sci Signal 2009;2:ra81.

-9 Hirt MN, Werner T, Indenbirken D, Alawi M, Demin P, Kunze AC, Stenzig J, Starbatty J, Hansen A, Fiedler J, Thum T, Eschenhagen T: Deciphering the microRNA signature of pathological cardiac hypertrophy by engineered heart tissue- and sequencing-technology. J Mol Cell Cardiol 2015;81:1-9.

$\checkmark 10$ McCutcheon CA, Orme RM, Scott DA, Davies MJ, McGlade DP: A comparison of dexmedetomidine versus conventional therapy for sedation and hemodynamic control during carotid endarterectomy performed under regional anesthesia. Anesth Analg 2006;102:668-675.

11 Ramsay MA, Luterman DL: Dexmedetomidine as a total intravenous anesthetic agent. Anesthesiology 2004;101:787-790.

-12 Sun Y, Jiang C, Jiang J, Qiu L: Dexmedetomidine protects mice against myocardium ischaemic/reperfusion injury by activating an AMPK/PI3K/Akt/eNOS pathway. Clin Exp Pharmacol Physiol 2017;44:946-953.

13 Si YN, Bao HG, Xu L, Wang XL, Shen Y, Wang JS, Yang XB: Dexmedetomidine protects against ischemia/ reperfusion injury in rat kidney. Eur Rev Med Pharmacol Sci 2014;18:1843-1851.

14 Paeschke N, von Haefen C, Endesfelder S, Sifringer M, Spies CD: Dexmedetomidine Prevents Lipopolysaccharide-Induced MicroRNA Expression in the Adult Rat Brain. Int J Mol Sci 2017;18.

15 Zhang Q, Wu D, Yang Y, Liu T, Liu H: Dexmedetomidine Alleviates Hyperoxia-Induced Acute Lung Injury via Inhibiting NLRP3 Inflammasome Activation. Cell Physiol Biochem 2017;42:1907-1919.

16 Wang Y, Wu S, Yu X, Zhou S, Ge M, Chi X, Cai J: Dexmedetomidine Protects Rat Liver against IschemiaReperfusion Injury Partly by the alpha2A-Adrenoceptor Subtype and the Mechanism Is Associated with the TLR4/NF-kappaB Pathway. Int J Mol Sci 2016;17 pii: E995.

17 Gencer B, Karaca T, Tufan HA, Kara S, Arikan S, Toman H, Karaboga I, Hanci V: The protective effects of dexmedetomidine against apoptosis in retinal ischemia/reperfusion injury in rats. Cutan Ocul Toxicol 2014;33:283-288.

18 Wang Q, She Y, Bi X, Zhao B, Ruan X, Tan Y: Dexmedetomidine Protects PC12 Cells from Lidocaine-Induced Cytotoxicity Through Downregulation of COL3A1 Mediated by miR-let-7b. DNA Cell Biol 2017;36:518-528.

19 Li N, Ouyang BS, Liu L, Lin CS, Xing DD, Liu J: Dexmedetomidine protected COPD-induced lung injury by regulating miRNA-146a. Bratisl Lek Listy 2016;117:539-542.

$>20$ Pritchard CC, Cheng HH, Tewari M: MicroRNA profiling: approaches and considerations. Nat Rev Genet 2012;13:358-369.

-21 Eminaga S, Christodoulou DC, Vigneault F, Church GM, Seidman JG: Quantification of microRNA expression with next-generation sequencing. Curr Protoc Mol Biol 2013;Chapter 4:Unit 4.17.

22 Joshi SR, Dhagia V, Gairhe S, Edwards JG, McMurtry IF, Gupte SA: MicroRNA-140 is elevated and mitofusin-1 is downregulated in the right ventricle of the Sugen5416/hypoxia/normoxia model of pulmonary arterial hypertension. Am J Physiol Heart Circ Physiol 2016;311:H689-698.

23 Karakas M, Schulte C, Appelbaum S, Ojeda F, Lackner KJ, Munzel T, Schnabel RB, Blankenberg S, Zeller T: Circulating microRNAs strongly predict cardiovascular death in patients with coronary artery diseaseresults from the large AtheroGene study. Eur Heart J 2017;38:516-523. 


\section{Cellular Physiology Cell Physiol Biochem 2018;50:552-568 \begin{tabular}{ll|l} 
and Biochemistry Published onlIne: 11 October 2018 & $\begin{array}{l}\text { (c) } 2018 \text { The Author(s). Published by S. Karger AG, Basel } \\
\text { www.karger.com/cpb }\end{array}$ \\
\hline
\end{tabular}}

Yang et al.: Dexmedetomidine and Circulating miRNA

-24 Li XD, Yang YJ, Wang LY, Qiao SB, Lu XF, Wu YJ, Xu B, Li HF, Gu DF: Elevated plasma miRNA-122, -140-3p, $-720,-2861$, and -3149 during early period of acute coronary syndrome are derived from peripheral blood mononuclear cells. PLoS One 2017;12:e0184256.

25 Li X, Yang Y, Wang L, Qiao S, Lu X, Wu Y, Xu B, Li H, Gu D: Plasma miR-122 and miR-3149 Potentially Novel Biomarkers for Acute Coronary Syndrome. PLoS One 2015;10:e0125430.

-26 Li J, Li Y, Jiao J, Wang J, Li Y, Qin D, Li P: Mitofusin 1 is negatively regulated by microRNA 140 in cardiomyocyte apoptosis. Mol Cell Biol 2014;34:1788-1799.

-27 Scrutinio D, Conserva F, Passantino A, Iacoviello M, Lagioia R, Gesualdo L: Circulating microRNA-150-5p as a novel biomarker for advanced heart failure: A genome-wide prospective study. J Heart Lung Transplant 2017;36:616-624.

-28 Bostjancic E, Zidar N, Glavac D: MicroRNA microarray expression profiling in human myocardial infarction. Dis Markers 2009;27:255-268.

29 Wang W, Sun G, Zhang L, Shi L, Zeng Y: Circulating microRNAs as novel potential biomarkers for early diagnosis of acute stroke in humans. J Stroke Cerebrovasc Dis 2014;23:2607-2613.

-30 He XM, Zheng YQ Liu SZ, Liu Y, He YZ, Zhou XY: Altered Plasma MicroRNAs as Novel Biomarkers for Arteriosclerosis Obliterans. J Atheroscler Thromb 2016;23:196-206.

-31 Ren XP, Wu J, Wang X, Sartor MA, Jones K, Qian J, Nicolaou P, Pritchard TJ, Fan GC: MicroRNA-320 is involved in the regulation of cardiac ischemia/reperfusion injury by targeting heat-shock protein 20 Circulation 2009;119:2357-2366.

-32 Thum T, Galuppo P, Wolf C, Fiedler J, Kneitz S, van Laake LW, Doevendans PA, Mummery CL, Borlak J, Haverich A, Gross C, Engelhardt S, Ertl G, Bauersachs J: MicroRNAs in the human heart: a clue to fetal gene reprogramming in heart failure. Circulation 2007;116:258-267.

-33 Jakob P, Kacprowski T, Briand-Schumacher S, Heg D, Klingenberg R, Stahli BE, Jaguszewski M, Rodondi N, Nanchen D, Raber L, Vogt P, Mach F, Windecker S, Volker U, Matter CM, Luscher TF, Landmesser U: Profiling and validation of circulating microRNAs for cardiovascular events in patients presenting with ST-segment elevation myocardial infarction. Eur Heart J 2017;38:511-515.

-34 Wong LL, Rademaker MT, Saw EL, Lew KS, Ellmers LJ, Charles CJ, Richards AM, Wang P: Identification of novel microRNAs in the sheep heart and their regulation in heart failure. Sci Rep 2017;7:8250.

-35 Goren Y, Kushnir M, Zafrir B, Tabak S, Lewis BS, Amir O: Serum levels of microRNAs in patients with heart failure. Eur J Heart Fail 2012;14:147-154.

-36 Sommariva E, D’Alessandra Y, Farina FM, Casella M, Cattaneo F, Catto V, Chiesa M, Stadiotti I, Brambilla S, Dello Russo A, Carbucicchio C, Vettor G, Riggio D, Sandri MT, Barbuti A, Vernillo G, Muratori M, Dal Ferro M, Sinagra G, Moimas S: MiR-320a as a Potential Novel Circulating Biomarker of Arrhythmogenic CardioMyopathy. 2017;7:4802.

-37 Vacchi-Suzzi C, Hahne F, Scheubel P, Marcellin M, Dubost V, Westphal M, Boeglen C, Buchmann-Moller S, Cheung MS, Cordier A, De Benedetto C, Deurinck M, Frei M, Moulin P, Oakeley E, Grenet O, Grevot A, Stull R, Theil D et al.: Heart structure-specific transcriptomic atlas reveals conserved microRNA-mRNA interactions. PLoS One 2013;8:e52442.

-38 Cakmak HA, Coskunpinar E, Ikitimur B, Barman HA, Karadag B, Tiryakioglu NO, Kahraman K, Vural VA: The prognostic value of circulating microRNAs in heart failure: preliminary results from a genome-wide expression study. J Cardiovasc Med (Hagerstown) 2015;16:431-437.

-39 Pan Z, Sun X, Shan H, Wang N, Wang J, Ren J, Feng S, Xie L, Lu C, Yuan Y, Zhang Y, Wang Y, Lu Y, Yang B: MicroRNA-101 inhibited postinfarct cardiac fibrosis and improved left ventricular compliance via the FBJ osteosarcoma oncogene/transforming growth factor-beta1 pathway. Circulation 2012;126:840-850.

40 Xiao L, He H, Ma L, Da M, Cheng S, Duan Y, Wang Q, Wu H, Song X, Duan W, Tian Z, Hou Y: Effects of miR29a and miR-101a Expression on Myocardial Interstitial Collagen Generation After Aerobic Exercise in Myocardial-infarcted Rats. Arch Med Res 2017;48:27-34.

-41 Wu D, Jiang H, Chen S, Zhang H: Inhibition of microRNA-101 attenuates hypoxia/reoxygenationinduced apoptosis through induction of autophagy in H9c2 cardiomyocytes. Mol Med Rep 2015;11:3988-3994.

-42 Sukma Dewi I, Hollander Z, Lam KK, McManus JW, Tebbutt SJ, Ng RT, Keown PA, McMaster RW, McManus BM, Gidlöf O, Öhman J: Association of Serum MiR-142-3p and MiR-101-3p Levels with Acute Cellular Rejection after Heart Transplantation. PLoS One 2017;12. 


\section{Cellular Physiology Cell Physiol Biochem 2018;50:552-568

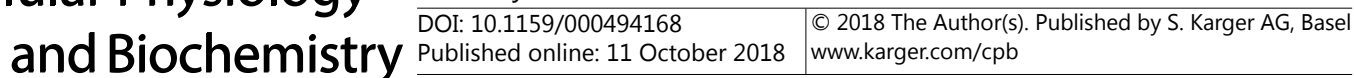

Yang et al.: Dexmedetomidine and Circulating miRNA

43 Lempiainen J, Finckenberg P, Mervaala EE, Storvik M, Kaivola J, Lindstedt K, Levijoki J, Mervaala EM: Dexmedetomidine preconditioning ameliorates kidney ischemia-reperfusion injury. Pharmacol Res Perspect 2014;2:e00045.

44 Xu Y, Zhang R, Li C, Yin X, Lv C, Wang Y, Zhao W, Zhang X: Dexmedetomidine attenuates acute lung injury induced by lipopolysaccharide in mouse through inhibition of MAPK pathway. Fundam Clin Pharmacol 2015;29:462-471.

45 Liao Z, Cao D, Han X, Liu C, Peng J, Zuo Z, Wang F, Li Y: Both JNK and P38 MAPK pathways participate in the protection by dexmedetomidine against isoflurane-induced neuroapoptosis in the hippocampus of neonatal rats. Brain Res Bull 2014;107:69-78.

-46 Wang Q, Tan Y, Zhang N, Xu Y, Wei W, She Y, Bi X, Zhao B, Ruan X: Dexmedetomidine inhibits activation of the MAPK pathway and protects PC12 and NG108-15 cells from lidocaine-induced cytotoxicity at its maximum safe dose. Biomed Pharmacother 2017;91:162-166.

47 Bao RF, Shu YJ, Hu YP, Wang XA, Zhang F, Liang HB, Ye YY, Li HF, Xiang SS, Weng H, Cao Y, Wu XS, Li ML, Wu WG, Zhang YJ, Jiang L, Dong Q, Liu YB: miR-101 targeting ZFX suppresses tumor proliferation and metastasis by regulating the MAPK/Erk and Smad pathways in gallbladder carcinoma. Oncotarget 2016;7:22339-22354.

48 Gao Y, Liu F, Fang L, Cai R, Zong C, Qi Y: Genkwanin inhibits proinflammatory mediators mainly through the regulation of miR-101/MKP-1/MAPK pathway in LPS-activated macrophages. PLoS One 2014;9:e96741.

-49 Yang J, Lu YW, Lu MM, Leng RX, Pan HF, Ye DQ: MicroRNA-101, mitogen-activated protein kinases and mitogen-activated protein kinases phosphatase-1 in systemic lupus erythematosus. Lupus 2013;22:115120.

50 Valente AJ, Yoshida T, Gardner JD, Somanna N, Delafontaine P, Chandrasekar B: Interleukin-17A stimulates cardiac fibroblast proliferation and migration via negative regulation of the dual-specificity phosphatase MKP-1/DUSP-1. Cell Signal 2012;24:560-568.

-51 Reforgiato MR, Milano G, Fabrias G, Casas J, Gasco P, Paroni R, Samaja M, Ghidoni R, Caretti A, Signorelli $\mathrm{P}$ : Inhibition of ceramide de novo synthesis as a postischemic strategy to reduce myocardial reperfusion injury. Basic Res Cardiol 2016;111:12.

52 Yue HW, Liu J, Liu PP, Li WJ, Chang F, Miao JY, Zhao J: Sphingosylphosphorylcholine protects cardiomyocytes against ischemic apoptosis via lipid raft/PTEN/Akt1/mTOR mediated autophagy. Biochim Biophys Acta 2015;1851:1186-1193.

53 Fan J, Wu BX, Crosson CE: Suppression of Acid Sphingomyelinase Protects the Retina from Ischemic Injury. Invest Ophthalmol Vis Sci 2016;57:4476-4484.

54 Lee HK, Jang JY, Yoo HS, Seong YH: Neuroprotective effect of phytoceramide against transient focal ischemia-induced brain damage in rats. Arch Pharm Res 2015;38:2241-2250. 